\title{
Early Phototherapy at Lower Total Serum Bilirubin Can Decrease Auditory Neuropathy Disorder
}

\author{
Tahereh Esmaeilnia ${ }^{1}$, Setareh Sagheb ${ }^{1,{ }^{*}}$, Mahdi Sepidarkish ${ }^{2}$, Sayyed Ourmazd Mohseni ${ }^{3}$, Atoosa \\ Moeinafshar ${ }^{4}$, Alireza Karimi-Yazdi ${ }^{5}$ and Mamak Shariat ${ }^{6}$ \\ ${ }^{1}$ Department of Pediatrics, Tehran University of Medical Sciences, Tehran, Iran \\ ${ }^{2}$ Department of Biostatistics and Epidemiology, Babol University of Medical Sciences, Babol, Iran \\ ${ }^{3}$ School of Molecular Sciences. Arizona State University, Tempe, United States \\ ${ }^{4}$ Tehran University of Medical Sciences, Tehran, Iran \\ ${ }^{5}$ Otorhinolaringology Research Center, Vali-e-Asr Hospital, Tehran University of Medical Sciences, Tehran, Iran \\ ${ }^{6}$ Breast Feeding Research Center, Vali-e-Asr Hospital, Tehran University of Medical Sciences, Tehran, Iran \\ "Corresponding author: Assistant Professor of Neonatology, Department of Neonatology, Tehran University of Medical Sciences, Tehran, Iran. Email: dr.ssagheb@yahoo.com
}

Received 2018 July 13; Revised 2018 December 14; Accepted 2018 December 22.

\begin{abstract}
Background: Neurologic dysfunctions, specifically hearing impairments due to hyperbilirubinemia are ranked among the main concerns in medicine.

Objectives: This study evaluates the prevalence of acute auditory neuropathy (AN) disorders in term neonates with mild to moderate hyperbilirubinemia and low bilirubin-induced neurological dysfunction (BIND) score and the roll of early treatment on reducing their incidence.

Methods: Fifty one term jaundice neonates with normal newborn exam and total serum bilirubin (TSB) $\geq 15 \mathrm{mg} / \mathrm{dL}$ after the third day of birth with normal otoacoustic emission (OAE) test were enrolled. The BIND score assessment for severity of neurologic dysfunction was done. Neonates with low BIND score were divided into two groups based on their TSB levels at the time of admission ( $15-18 \mathrm{mg} / \mathrm{dL}$ : Mild-moderate hyperbilirubinemia and $\geq 19 \mathrm{mg} / \mathrm{dL}$ : Severe hyperbilirubinemia). ABR was performed within the first 12 hours of their admission and the second ABR was performed before discharge.

Results: The $30.60 \%$ of neonates with TSB $<19 \mathrm{mg} / \mathrm{dl}$ had abnormal ABR. Latency of V, III waves and interpeak interval latency of I-III, $\mathrm{I}-\mathrm{V}$ waves were detected. Mean latency of wave V and I-V interpeak latency after phototherapy were significantly decreased compared to pre-treatment $(\mathrm{P}<0.001)$.

Conclusions: With the prevalence of auditory neuropathy (AN) at lower TSB concentrations (that have traditionally been considered safe) it can be concluded that more attention should be given to this group despite the absence of neurologic signs, and it also shows the sensitivity of early phototherapy to lower bilirubin level.
\end{abstract}

Keywords: Acute Bilirubin Encephalopathy, ABR, Hyperbilirubinemia, BIND Score, Auditory Neuropathy Disorder

\section{Background}

Despite rapid advances in neonatal care management, severe hyperbilirubinemia is still one of the most common problems during neonatal period that may lead to acute and chronic bilirubin encephalopathy (1). In developed countries, kernicterus incidence in term infants counts for 1 in 30,000 to 1 in 200,000 live births (2). Bilirubininduced neurological dysfunction(BIND) hosts a spectrum of neurological dysfunctions including acute bilirubin encephalopathy (ABE) and isolated auditory dysfunctions that lead to neurodevelopmental disabilities $(3,4)$. There is no accurate total serum bilirubin (TSB) threshold for identifying BIND and kernicterus $(5,6)$.
The scoring system of bilirubin-induced neurologic dysfunction can be considered as a useful clinical tool for diagnosing term neonates with acute bilirubin encephalopathy who require more aggressive treatment (7, 8).

Previous studies suggested that subtle bilirubin encephalopathy leads to central auditory system damage and auditory neuropathy $(9,10)$ with different presentations from speech disorders to complete deafness, which needs cochlear implantation $(6,7,11)$.

An abnormal or absent auditory brainstem evoked response (ABR) with a normal otoacoustic emission test (OAE) is considered as auditory neuropathy (7-12). Transient or lasting ABR abnormalities occur in $35 \%$ of neonates 
with TB levels $>20 \mathrm{mg} / \mathrm{dL}$. Previous studies suggested that performing ABR is crucial for infants with severe hyperbilirubinemia (9).

The abnormality in ABR results consists of wave I and III latencies, wave $\mathrm{V}$ mean latency, inter peak interval of III-V, I-III, I-V waves, or lack of each wave.

Absent or abnormal wave I is considered as auditory nerve damage and in waves III and V is damaged in the brainstem nuclei $(13,14)$.

Limited information is available about the prevalence rate of hearing loss due to acute auditory neuropathy at lower hyperbilirubinemia (10). Score of 1 - 3 in BIND score is considered as subtle signs of ABE. An abnormal ABR in this group is suggestive of bilirubin neurotoxicity which is considered as moderate $\mathrm{ABE}$ (7).

\section{Objectives}

The aim of this study was to evaluate whether mild to moderate hyperbilirubinemia in term neonates with low BIND score (score of 1 - 3) is associated with acute auditory neuropathy disorder and also evaluate the roll of early treatment in reducing incidence of acute auditory neuropathy in this group.

\section{Methods}

\subsection{Study Design}

This present study was a cross- sectional study performed at the Vali-e-Asr Hospital of Tehran University of Medical Sciences (TUMS) from August 2016 to November 2017 on all term newborns (with the gestational ages of $\geq 37$ weeks) who were admitted to the neonatal ward due to hyperbilirubinemia with TBS of $\geq 15 \mathrm{mg} / \mathrm{dL}$ after the third day of birth with normal OAE test. Gestational age of newborns was determined according to the first day of the mother's last menstrual period (by the mother's statement) and the first antenatal ultra-sonographic estimation.

Exclusion criteria: (1) infants born to mothers with chorioamnionitis; (2) craniofacial malformations; (3) preterm infants; (4) neonates with asphyxia; (5) early onset sepsis with positive blood culture; (6) anomalies of the nervous system; (7) positive family history of hearing loss; (8) history of using of ototoxic medicine; (9) drugs affecting the bonding of bilirubin; (10) direct hyperbilirubinemia; (11) chromosomal disorders; (12) hyperbilirubinemia in the first 24 hours of life; (13) infants whose mothers had a history of diabetes.

This study was approved by the Research and Medical Ethics Committee of Tehran University of Medical Sciences in accordance with the Helsinki Declaration and written consent was obtained from parents before enrollment.

The neonates were divided into two groups based on their total serum bilirubin (TSB) levels at time of admission (TSB 15 - $18 \mathrm{mg} / \mathrm{dL}$ : Mild-moderate hyperbilirubinemia and TSB $\geq 19 \mathrm{mg} / \mathrm{dL}$ : Severe hyperbilirubinemia). All neonates were examined by a resident and a fellow of neonatology at the time of admission and signs of the nervous system involvement were recorded based on BIND score (7). Score of 1 - 3 in BIND score was considered as subtle sign of ABE.

Management of hyperbilirubinemia was performed based on the American Academy of Pediatrics (AAP) guidelines (15). Routine tests for evaluation of jaundice including complete blood count (CBC), direct Coombs test, reticulocyte count, glucose-6-phosphate dehydrogenase (G6PD), peripheral smear, blood group determination and Rh typing of mother and neonate and thyroid function tests were performed in all cases. Serum bilirubin was measured with colorimetric methods (diazotized sulfanilic acid reaction, Roche Diagnostics GmbH, Mannheim, Germany). CBC was calculated by the automated hematology analyzer XE-1200 (System, Japan).

ABR was performed within the first 12 hours of admission and the second ABR was performed after treatment and before discharge from the hospital. All of the neonates had been sedated by oral chlorate hydrate, $50 \mathrm{mg}$ per $\mathrm{kg}$ of body weight half an hour before the test. Four electrodes were applied as follows: Two active ones were placed on mastoid bones, the reference electrode on vertex, and the ground electrode on the forehead.

An auditory evoked potential system (Charter ICS, Denmark) was used to record ABR. A click type stimulus was used twice with rarefaction polarity and intensity of 35 $80 \mathrm{~dB}$ nHL, under 21/1 pulse which was set based on 1500 trials and analysis time of 10 - 15 ms. All audiometric tests were performed by a trained audiologist, blinded to the study procedures. Measurable and comparable variables consisted of mean latency of V, III and I waves, interpeak interval of I-III, III-V, I-V waves and no waves.

The prevalence of abnormal ABR in the range of various changes of bilirubin level and ABR changes before and after treatment were compared.

\subsection{Statistical Analysis}

Categorical and continuous variables were summarized as number (\%) and mean $\pm \mathrm{SD}$. Continuous variables were analyzed using student $t$-test. Categorical variables were analyzed using chi-squared and Fisher exact test. Logistic regression analyses were performed to calculate the Odds ratio with 95\% confidence interval for the association of serum bilirubin and ABR. Statistical analyses were per- 
formed using Stata 11 (Stata Corporation, College Station, TX).

\section{Results}

A total of 85 term neonates with jaundice were admitted to our hospital, of which 51 fulfilled the eligibile criteria. The mean \pm SD birth weight and gestational age for the neonates were $3405 \pm 496.58 \mathrm{~g}$ and $38.20 \pm 0.66$ weeks, respectively. The study group included 23 (45.1\%) boys and 28 (54.9\%) girls. Exchange transfusion was performed on 10 (19.6\%) neonates. Of these 51 neonates, 15 (29.4\%) had abnormal ABR and 36 (70.6\%) had normal ABR. All neonates had negative direct Coombs, and 48 (94.1\%) neonates had sufficient G6PD. 17 (33.3\%) neonates had ABO incompatibility and four (26.7\%) of them had TSB $\geq 19 \mathrm{mg} / \mathrm{dL} .5$ (9.8\%) neonates had Rh incompatibility and 4 (11.1\%) had TSB $<19$ $\mathrm{mg} / \mathrm{dL}$.

The mean \pm SD TSB was $19.22 \pm 3.99 \mathrm{mg} / \mathrm{dL}$ and 15 (29.4\%) neonates had TSB above $19 \mathrm{mg} / \mathrm{dL}$. The mean \pm SD hospital stay in neonates with TSB $\geq 19 \mathrm{mg} / \mathrm{dL}$ was 4.60 \pm 5.46 which was significantly higher than $2.81 \pm 0.46$ in neonates with TSB $<19 \mathrm{mg} / \mathrm{dL}(\mathrm{P}<0.001)$. There was no significant difference between neonates with TSB $\geq 19$ $\mathrm{mg} / \mathrm{dL}$ and $<19 \mathrm{mg} / \mathrm{dL}$ regarding birth weight, exclusive breast feeding, gestational age, blood group and Rh incompatibility, and mean of hemoglobin (Table 1). The prevalence of abnormal ABR was $26.7 \%$ in neonates with TSB $\geq 19$ $\mathrm{mg} / \mathrm{dL}$, while the prevalence of abnormal ABR was $30.60 \%$ in neonates with TSB $<19 \mathrm{mg} / \mathrm{dL}$ (OR: 0.82, CI 95\%:0.21 - 3.17, $\mathrm{P}=0.781$ ). Using multiple logistic regression analysis, the odds ratio for the association between abnormal ABR and bilirubin level was estimated about 0.46 (CI 95\%: 0.03 - 6.74, $\mathrm{P}=0.571$ ).

The prevalence of abnormal ABR in the range of various changes of bilirubin level is shown in Table 2 .

The mean latency of $\mathrm{V}$ and III waves and interpeak interval of I-III and I-V waves before and after phototherapy in neonates with abnormal ABR is shown in Table 3.

Mean latency of wave $\mathrm{V}$ and $\mathrm{I}-\mathrm{V}$ interpeak latency after phototherapy was significantly decreased compared to pre-treatment $(\mathrm{P}<0.001)$. There was no significant difference regarding the mean latency of wave III and I-III interpeak interval latency before and after treatment.

\section{Discussion}

Neurologic dysfunction especially hearing impairments due to hyperbilirubinemia are still one of the main concerns in medicine. These children often experience delayed development of speech and cognitive skills which may lead to learning disabilities and behavior problems $(16,17)$. Performing ABR during hyperbilirubinemia was suggested to identify auditory neuropathy by previous studies $(9,10)$. In this study, we investigated the prevalence of auditory neuropathy diagnosed with abnormal ABR in term neonates who had mild to moderate hyperbilirubinemia with low BIND score.

In this study, $29.4 \%$ of jaundiced newborns had abnormal ABR. In Akinpelu et al.'s study, 35\% of neonates with TBS $>20 \mathrm{mg} / \mathrm{dL}$ had abnormal ABR(9). In Saluja et al.'s study of 13 neonates with severe hyperbilirubinemia requiring exchange transfusion, $46 \%$ had bilateral ABR abnormalities (14). In their study, the correlation between peaks of TBS and abnormalities in ABR was not evaluated. Zamiri Abdollahi et al. reported $26.8 \%$ of neonates with TBS $>20$ $\mathrm{mg} / \mathrm{dL}$ requiring exchange transfusion had abnormal ABR (18). Previous studies focused on the association between severe hyperbilirubinemia and abnormal ABR. It should be noted that there is no exact TSB threshold for identifying auditory dysfunctions. Hearing loss was diagnosed in 10\% - 37.5\% of neonates with hyperbilirubinemia with a TBS < $20 \mathrm{mg} / \mathrm{dL}$ (19-21). This was also included in our study. In present study, $26.7 \%$ of neonates with TSB $\geq 19 \mathrm{mg} / \mathrm{dL}$ had abnormal ABR, while this was $30.60 \%$ in neonates with TSB $<19 \mathrm{mg} / \mathrm{dL}$.

Although hemolysis is considered as a risk factor for $A B E$ (15), it had no effect on the prevalence of hearing loss between these subgroups. In this study, the prevalence of abnormal ABR in changes of bilirubin were evaluated. Although the prevalence of abnormal ABR is not statistically significant at any of the cutting points, it is noticeable at each cut point. At lower cut points, the prevalence of abnormal ABR was still high. Despite the high prevalence of isolated AN in our study; unfortunately, the small number of neonates in each group precludes a meaningful comparison between these subgroups for determining the exact TSB threshold for identifying AN in neonates with mild to moderate hyperbilirubinemia.

In our study, latency of V and III waves and interpeak interval latency of I-III and I-V waves were detected. This was also ireported in previous studies $(13,14,19,22)$, at the same time, prolongation of wave I latency was not detected. These are quite in agreement with Sharma et al.'s (23) and Salehi et al.'s (24) studies who observed that early prolongation of the latency of wave III and V and interpeak latency of wave I-III and I-V happened in moderate hyperbilirubinemia and any increase in wave I latency was diagnosed later especially during severe hyperbilirubinemia.

In this study, variables of mean latency of $\mathrm{V}$ and III waves and interpeak interval of I-III and I-V waves before and after phototherapy in neonates with abnormal ABR were evaluated and mean latency of wave $\mathrm{V}$ and $\mathrm{I}-\mathrm{V}$ in- 


\begin{tabular}{|c|c|c|c|}
\hline Variable & Neonates with Bilirubin $\geq 19 \mathrm{mg} / \mathrm{dL}(\mathrm{N}=15)$ & Neonates with Bilirubin $<19 \mathrm{mg} / \mathrm{dL}(\mathrm{N}=36)$ & P Value \\
\hline Gestational age, wk & $38.40 \pm 0.82$ & $38.11 \pm 0.57$ & 0.232 \\
\hline Birth weight, $\mathrm{g}$ & $3258.00 \pm 399.23$ & $3466.25 \pm 524.79$ & 0.175 \\
\hline Hemoglobin & $16.62 \pm 2.13$ & $15.71 \pm 2.11$ & 0.168 \\
\hline NICU admission, $d$ & $4.60 \pm 0.63$ & $2.81 \pm 0.46$ & $<0.001$ \\
\hline Gender, boy/girl & $3 / 12$ & $20 / 16$ & 0.030 \\
\hline Blood transfusion & & & $<0.001$ \\
\hline Yes & $10(66.7)$ & $0(0)$ & \\
\hline No & $5(33.3)$ & $36(100)$ & \\
\hline Mode of delivery & & & 0.013 \\
\hline NVD & $6(40)$ & $3(8.3)$ & \\
\hline $\mathrm{C} / \mathrm{S}$ & $9(60)$ & $33(91.7)$ & \\
\hline Exclusive breastfeeding & & & 0.305 \\
\hline Yes & $15(100)$ & $31(86.1)$ & \\
\hline No & $0(0)$ & $5(13.9)$ & \\
\hline \multicolumn{4}{|c|}{ Abbreviations: C/S, cesarean section; NVD, normal vaginal delivery. } \\
\hline Variable & Abnormal ABR, No. (\%) & Normal ABR, No. (\%) & PValue \\
\hline $\mathrm{TSB} \geq 19, \mathrm{mg} / \mathrm{dL}$ & $4(26.7)$ & $11(73.3)$ & \multirow{2}{*}{0.781} \\
\hline TSB $<19, \mathbf{m g} / \mathrm{dL}$ & $11(30.6)$ & $15(69.4)$ & \\
\hline $\mathrm{TSB} \geq \mathbf{1 8}, \mathbf{m g} / \mathrm{dL}$ & $5(23.8)$ & $16(76.2)$ & \multirow{2}{*}{0.463} \\
\hline TSB $<18, \mathrm{mg} / \mathrm{dL}$ & $10(33.3)$ & $20(66.7)$ & \\
\hline $\mathrm{TSB} \geq 17, \mathrm{mg} / \mathrm{dL}$ & $7(24.1)$ & $22(75.9)$ & \multirow{2}{*}{0.343} \\
\hline TSB $<17, \mathbf{m g} / \mathbf{d L}$ & $8(36.4)$ & $14(63.6)$ & \\
\hline $\mathrm{TSB} \geq 16, \mathrm{mg} / \mathrm{dL}$ & $11(27.5)$ & $29(72.5)$ & \multirow{2}{*}{0.711} \\
\hline $\mathrm{TSB}<16, \mathrm{mg} / \mathrm{dL}$ & $4(36.4)$ & $7(63.6)$ & \\
\hline
\end{tabular}

Abbreviations: TSB, total serum bilirubin.

Table 3. Comparison of Mean \pm SD Latency of V, III Waves and Interpeak Interval of I-III, I-V Waves Before and After Phototherapy in Neonates with Abnormal ABR

\begin{tabular}{lcc}
\hline Latency/Interpeak Wave & Before Treatment & After Treatment \\
\hline Prolonged latency of wave III & $4.91 \pm 0.25$ & $4.88 \pm 0.28$ \\
Prolonged latency of wave V & $7.66 \pm 0.29$ & $7.09 \pm 0.19$ \\
Prolonged lnterpeak latencies I-III & $2.99 \pm 0.22$ & $3.10 \pm 0.33$ \\
Prolonged Interpeak latencies I-V & $5.54 \pm 0.30$ & 0.726 \\
\hline
\end{tabular}

terpeak latency after phototherapy were significantly decreased compared to pre-treatment $(\mathrm{P}<0.001)$.

Previous studies demonstrated that auditory neuropathy due to hyperbilirubinemia could be reversible after treatment (phototherapy or exchange transfusion) by the end of the 3rd month of age (24-26).

We were focused on the possibility of hearing damage at lower level of hyperbilirubinemia and evaluated effects of early treatment on auditory neuropathy before their discharge from the hospital. It seems that auditory neuropathy may occur at lower TBS concentrations that are widely used for therapeutic interventions.

Despite high incidence of acute auditory neuropathy among neonates with mild to moderate hyperbilirubine- 
mia in our study, with early intervention change in ABR waves could be reversible.

It should be stated that performing the ABR test to diagnose infants with mild hyperbilirubinemia susceptible to auditory neuropathy disorder should be necessary for early identification and also early phototherapy .This may improve the long term neurodevelopmental outcome of these infants especially speech and language disorders during their childhood. This is specifically valuable in countries such as Iran in which the prevalence of G6PD deficiency is considerably high.

\subsection{Limitation}

Despite the high prevalence of isolated AN in our study; unfortunately, the small number of neonates in each group precludes a meaningful comparison between these subgroups for determining the exact TSB threshold in order to identify AN in neonates with mild to moderate hyperbilirubinemia. As this is a referral center and many patients are referred from other cities, unfortunately, we could not repeat ABR test at the third month. It will be more conclusive to perform this investigation in multicenter studies.

\subsection{Conclusions}

With the prevalence of auditory neuropathy (AN) at lower TSB concentrations (that have traditionally been considered safe) it can be concluded that more attention should be given to this group despite the absence of neurologic signs, and it also shows the sensitivity of early phototherapy to lower bilirubin level.

\section{Footnotes}

Authors' Contribution: Tahereh Esmaeilnia conceived paper, critically revised of manuscript and approved final version. Setareh Sagheb participated in study design, oversaw data collection, interpretation of data and revision of manuscript and approved final version. Sayyed Ourmazd Mohseni conceived paper, participated in study design, wrote manuscript, critically revised of manuscript and approved final version. Mahdi Sepidarkish participated in study design, conducted data analysis and interpretation, critically revised manuscript and approved final version. Atoosa Moeinafshar participated in study design, interpretation of data and revision of manuscript and approved final version. Alireza Karimi-Yazdi participated in study design, interpretation of data and revision of manuscript and approved final version. Mamak Shariat participated in study design, interpretation of data and revision of manuscript and approved final version.
Conflict of Interests: No financial interests related to the material in the manuscript.

Ethical Considerations:

Ethical Code No: IR.TUMS.REC.1395.2263.

Funding/Support: This work was supported by Tehran University of Medical Sciences [grant numbers 94-02-9129545].

\section{References}

1. Shapiro SM. Kernicterus. In: Stevenson DK, Maisels MJ, Watchko JF, editors. Care of the jaundiced neonate. New York: McGraw-Hill; 2012. p. 229-42.

2. Maisels MJ, Newman TB. Prevention, screening, and postnatal management of neonatal hyperbilirubinemia. In: Stevenson DK, Maisels MJ, Watchko JF, editors. Care of the jaundiced neonate. New York: McGraw Hill; 2012. p. 175-94.

3. Shapiro SM. Chronic bilirubin encephalopathy: Diagnosis and outcome. Semin Fetal Neonatal Med. 2010;15(3):157-63. doi: 10.1016/j.siny.2009.12.004. [PubMed: 20116355].

4. Johnson L, Bhutani VK. The clinical syndrome of bilirubin-induced neurologic dysfunction. Semin Perinatol. 2011;35(3):101-13. doi: 10.1053/j.semperi.2011.02.003. [PubMed: 21641482].

5. Watchko JF, Tiribelli C. Bilirubin-induced neurologic damagemechanisms and management approaches. $N$ Engl $J$ Med. 2013;369(21):2021-30. doi: 10.1056/NEJMra1308124. [PubMed: 24256380].

6. Ahlfors CE. Predicting bilirubin neurotoxicity in jaundiced newborns. Curr Opin Pediatr. 2010;22(2):129-33. doi: 10.1097/MOP.ob013e328336eb28. [PubMed: 20125026].

7. Johnson L, Bhutani VK, Karp K, Sivieri EM, Shapiro SM. Clinical report from the pilot USA kernicterus registry (1992 to 2004).JPerinatol. 2009;29 Suppl 1:S25-45. doi:10.1038/jp.2008.211. [PubMed: 19177057].

8. Gamaleldin R, Iskander I, Seoud I, Aboraya H, Aravkin A, Sampson PD, et al. Risk factors for neurotoxicity in newborns with severe neonatal hyperbilirubinemia. Pediatrics. 2011;128(4):e925-31. doi: 10.1542/peds.2011-0206. [PubMed: 21911352]. [PubMed Central: PMC3182847].

9. Akinpelu OV, Waissbluth S, Daniel SJ.Auditory risk of hyperbilirubinemia in term newborns: A systematic review. Int J Pediatr Otorhinolaryngol. 2013;77(6):898-905. doi: 10.1016/j.ijporl.2013.03.029. [PubMed: 23642487].

10. Smith CM, Barnes GP, Jacobson CA, Oelberg DG. Auditory brainstem response detects early bilirubin neurotoxicity at low indirect bilirubin values. J Perinatol. 2004;24(11):730-2. doi: 10.1038/sj.jp.7211164. [PubMed: 15510103].

11. Shapiro SM, Popelka GR. Auditory impairment in infants at risk for bilirubin-induced neurologic dysfunction. Semin Perinatol. 2011;35(3):162-70. doi: 10.1053/j.semperi.2011.02.011. [PubMed: 21641490].

12. Akman I, Ozek E, Kulekci S, Turkdogan D, Cebeci D, Akdas F. Auditory neuropathy in hyperbilirubinemia: Is there a correlation between serum bilirubin, neuron-specific enolase levels and auditory neuropathy? Int J Audiol. 2004;43(9):516-22. [PubMed: 15726842].

13. Shapiro SM. Definition of the clinical spectrum of kernicterus and bilirubin-induced neurologic dysfunction (BIND). J Perinatol. 2005;25(1):54-9. doi: 10.1038/sj.jp.7211157. [PubMed: 15578034].

14. Saluja S, Agarwal A, Kler N, Amin S. Auditory neuropathy spectrum disorder in late preterm and term infants with severe jaundice. Int J Pediatr Otorhinolaryngol. 2010;74(11):1292-7. doi: 10.1016/j.ijporl.2010.08.007. [PubMed: 20832127]. [PubMed Central: PMC2962441]. 
15. American Academy of Pediatrics Subcommittee on Hyperbilirubinemia. Management of hyperbilirubinemia in the newborn infant 35 or more weeks of gestation. Pediatrics. 2004;114(1):297-316. [PubMed: 15231951].

16. Burke MJ, Shenton RC, Taylor MJ. The economics of screening infants at risk of hearing impairment: An international analysis. Int J Pediatr Otorhinolaryngol. 2012;76(2):212-8. doi: 10.1016/j.ijporl.2011.11.004. [PubMed: 22129917].

17. Amin SB, Prinzing D, Myers G. Hyperbilirubinemia and language delay in premature infants. Pediatrics. 2009;123(1):327-31. doi: 10.1542/peds.2007-3723. [PubMed: 19117899]. [PubMed Central: PMC3264948].

18. Zamiri Abdollahi F, Ahmadi T, Manchaiah V, Lotfi Y. Auditory brainstem response improvements in hyperbillirubinemic infants.JAudiol Otol. 2016;20(1):13-6. doi: 10.7874/jao.2016.20.1.13. [PubMed: 27144228]. [PubMed Central: PMC4853896].

19. Jiang ZD, Liu TT, Chen C. Brainstem auditory electrophysiology is supressed in term neonates with hyperbilirubinemia. Eur J Paediatr Neurol. 2014;18(2):193-200. doi: 10.1016/j.ejpn.2013.11.004. [PubMed: 24309481].

20. Wong V, Chen WX, Wong KY. Short- and long-term outcome of severe neonatal nonhemolytic hyperbilirubinemia. J Child Neurol. 2006;21(4):309-15. doi: 10.1177/08830738060210040301. [PubMed: 16900927].
21. Esmaeilnia T, Shariat M, Ebrahim B, Moghimpour Bijani F. Relationship between auditory brainstem response and neonatal hyperbilirubinemia before and after treatment. Aud Vest Res. 2015;24(4):210-6.

22. Jiang ZD, Wilkinson AR. Impaired function of the auditory brainstem in term neonates with hyperbilirubinemia. Brain Dev. 2014;36(3):2128. doi: 10.1016/j.braindev.2013.03.003. [PubMed: 23587715].

23. Sharma R, Grover N, Sankhyan N, Sharma ML. Auditory brainstem responses in neonatal hyperbilirubinemia and effect of therapy. Indian J Otolaryngol Head Neck Surg. 2006;58(4):340-2. doi: 10.1007/BF03049585. [PubMed: 23120340]. [PubMed Central: PMC3450378].

24. Salehi N, Bagheri F, Ramezani Farkhani H. Effects of hyperbilirubinemia on auditory brainstem response of neonates treated with phototherapy. Iran J Otorhinolaryngol. 2016;28(84):23-9. [PubMed: 26878000]. [PubMed Central: PMC4735613].

25. Vinodh M, Ambikapathy P, Aravind MA, Ganesh J. Reversibility of brainstem evoked response audiometry abnormalities at 3 months in term newborns with hyperbilirubinemia. Indian Pediatr. 2014;51(2):134-5. [PubMed: 24277970].

26. Krishnegowda S, Srinivasiah S, Ramachandra K, Keshavamurthy S. A study of brainstem evoked response audiometry in term neonates with hyperbilirubinemia. Int J Contemp Pediatr. 2016:583-6. doi: 10.18203/2349-3291.ijcp20161043. 